\title{
A 35-Year-old Man With New Onset Blindness
}

\author{
Cecilia Kelly, MD, Claire Raab, MD and Aimee Lee, MSIV
}

\section{Case Report}

A 35 year-old homosexual male from Trinidad with no known past medical history presented with a complaint of new-onset blindness. He stated that his vision had become increasingly "dim" to the point that at presentation, he had minimal light perception in either eye. This decrease in vision had occurred over the course of a week. He denied any trauma, eye pain, headache, nausea or vomiting.

On review of systems, the patient reported bilateral lower extremity numbness and tingling as well as left lower extremity weakness that had been present for approximately six months. He attributed these neurologic symptoms to a car accident one-year prior. Over these six months, he was additionally complaining of intermittent night sweats and chills, patchy hair loss on his scalp, and facial acne. Further questioning revealed a history of unprotected anal intercourse with several male partners. He had not undergone any testing for sexually transmitted diseases in the past. He had not seen a doctor for over 10 years.

Physical examination was significant for patchy alopecia of the scalp, perioral ulcerating lesions, and four nonerythematous soft macular lesions, approximately $2 \mathrm{~mm} \times 3 \mathrm{~mm}$, on the left cheek and chin. Neurological examination revealed normal cranial nerves except for in the pupilary exam, which displayed small pupils bilaterally that were reactive to accommodation, but did not react when exposed to light. Strength was decreased to $4 / 5$ in the right lower extremity in all muscle groups and $3 / 5$ in the muscle groups of the left lower extremity, with an associated left-sided foot drop. Lower extremity reflexes were absent bilaterally.

Laboratory studies revealed a profound lymphopenia. A rapid human immunodeficiency virus (HIV) test was positive

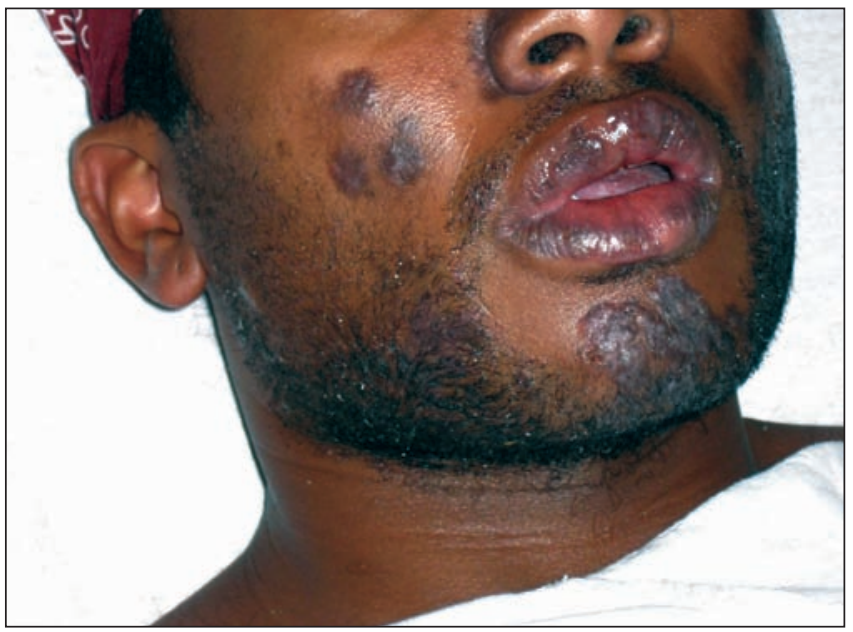

Figure 1. Small rubbery gummatous lesions seen on the right cheek, chin, and lips in a patient with tertiary syphilis. with western blot confirmation. CD4+ count was 40 cells/ $\mathrm{mm}^{3}$. VDRF, RPR, and FTA-ABS (venereal disease research laboratory, rapid plasma reagin, fluorescent treponemal antibody absorption test; FTA-ABS respectively) were positive as well. With these neurological symptoms in the setting of a new diagnosis of acquired immunodeficiency syndrome (AIDS) and syphilis, lumbar puncture was performed to look for infection. The results of cerebrospinal fluid analysis showed an elevated protein level with a normal glucose, lymphocytic pleocytosis, and a reactive VDRL serology, findings that were all consistent with neurosyphilis.

Consequently, the patient was initiated on a 3 week course of intravenous penicillin $\mathrm{G}$. He was able to recover partial vision; however his foot drop and numbness persisted, requiring the use of a cane for support with mobility.

\section{Discussion}

Syphilis is a treatable sexually transmitted infection caused by the spirochete Treponema pallidum. The natural history of this infection can be divided into early and late processes. Early syphilis includes primary, secondary, and latent infections. If left untreated, these can progress to tertiary syphilis, the late process of the disease which is no longer curable. Late syphilis occurs after a latent period of one to thirty years following initial infection.

Tertiary syphilis is characterized by cardiovascular, dermatologic, and neurologic involvement. Patients develop chronic inflammation of the thoracic aorta resulting in aortic dilatation and aortic valve regurgitation. These manifestations can present as a murmur, usually that of aortic insufficiency, and can result in left-sided heart failure. ${ }^{1}$ Granuloma formations, known as gummas, are specific to syphilis and found in dermal, visceral, or bony areas. Our patient presented with gummas on his face that he mistook for acne (Figure 1). Gummas can present as ulcerating lesions on the skin or even as mass lesions.

General paresis and personality changes progressing to dementia are also characteristic of neurologic syphilis. ${ }^{2}$ Syphilitic involvement of the central nervous system can cause damage to the posterior columns and dorsal roots of the spinal cord, known as the syndrome of tabes dorsalis. This involvement results in limb paresthesias, episodic nausea and vomiting, absent lower extremity reflexes, and decreased vibratory and positional awareness. On physical exam patients may present with the classic finding of Argyll-Robinson pupils, which refer to the condition where the pupils are able to accommodate but unable to constrict to light or painful stimuli. Other findings include dysarthria and intention tremors. ${ }^{2}$ Many of these clinical signs, including gummas, paresthesias, paresis, and Argyll-Robinson pupils, were seen in our patient. 
Before the use of antibiotics, tertiary syphilis occurred in 20-40\% of all individuals that had been infected with the disease. ${ }^{2}$ In the United States, the Center for Disease Control and Prevention (CDC) reports that the rates of neurosyphilis ranged from 5.5-6.0 cases per 100,000 infected individuals in 2004. ${ }^{1}$ In 2007, primary and secondary syphilis prevalence in Philadelphia, Camden, and Wilmington areas was 3.5\% with 205 new cases reported. $^{2}$

Neurosyphilis in HIV/AIDS patients has been recognized and reported since the beginning of the HIV/AIDS epidemic. ${ }^{1}$ Symptomatic neurosyphilis is a rare manifestation of syphilis in the United States, due to the near complete eradication in the 1940s following the introduction of penicillin. However, neurosyphilis began to appear again in the 1980s in patients infected with HIV. In the early 2000s, the incidence of neurosyphilis began to rise dramatically, primarily in men who have sex with men (MSM). The CDC conducted a review of these cases, reporting an estimated $1.7 \%$ risk of symptomatic early neurosyphilis in HIV-positive MSM, and an estimated $0.5 \%$ risk of persistent symptoms six months after treatment. ${ }^{2}$

\section{Conclusion}

Because of the increasing incidence, clinicians should have a high index of suspicion for syphilis in HIV-positive MSM presenting with meningismus, or motor or sensory cranial nerve deficits. As seen in this case, the clinician must be sure to rule out HIV/ AIDS in a MSM who is presenting with neurosyphilis.

\section{References}

1. Katz DA, Berger JR. Neurosyphilis in acquired immunodeficiency syndrome Arch Neurol 1989;46:895--8.

2. Lee MA, Aynalem G, Kerndt P. Symptomatic Early Neurosyphilis Among HIV-Positive Men Who Have Sex With Men Four Cities, United States. JAMA 2007;298(7):732-734.

"Great Pyramids of Giza, Egypt", photograph by Sameh Gaballa, MD

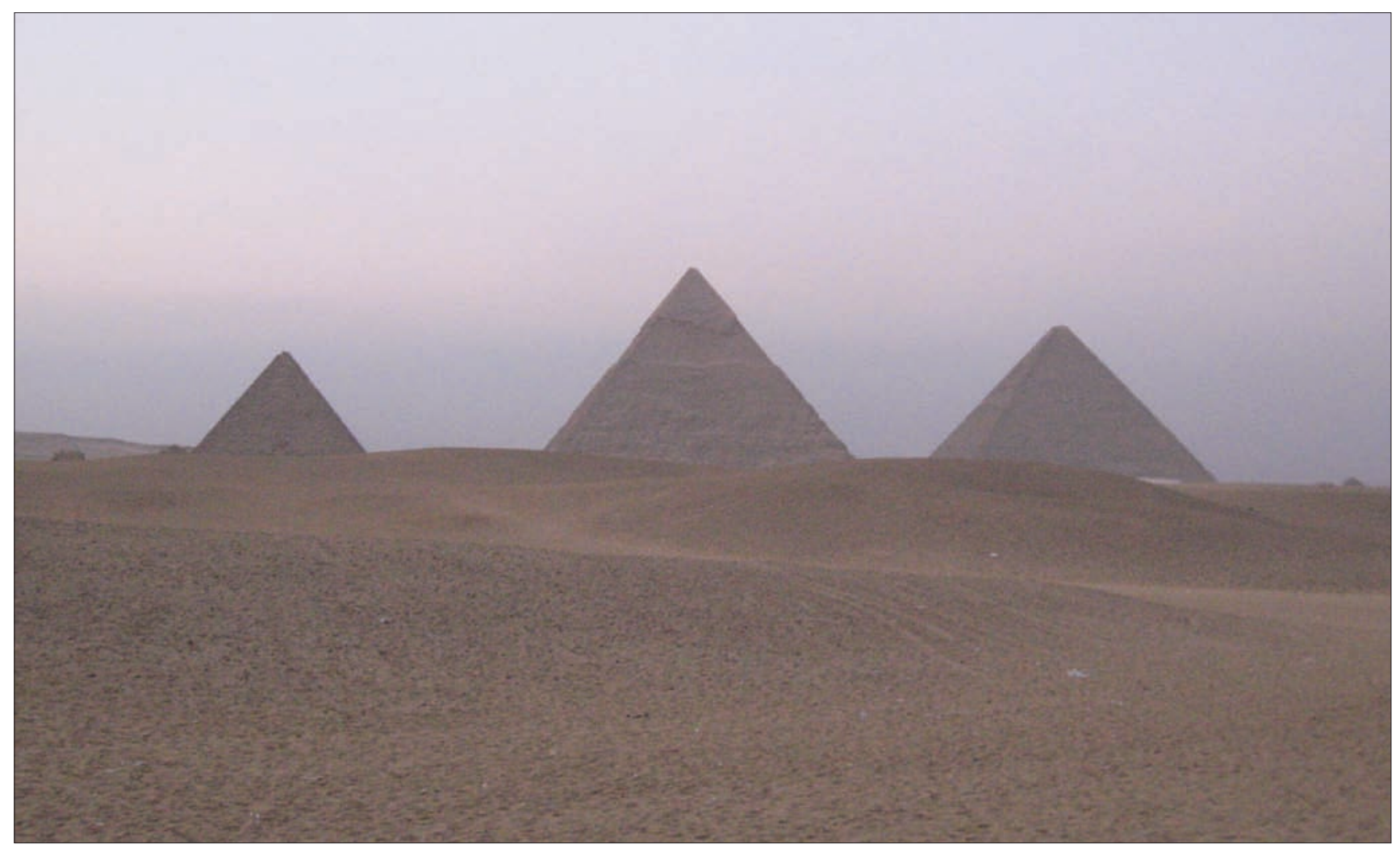

